\title{
O PROJETO E A FABRICAÇÃo POR MANUFATURA AdITIVA: CASO dOS PROTETORES FACIAIS
}

Alexandre Crepory Abbot de Oliveira (alex.crepory@gmail.com) - PPGEE, Universidade de Brasília Tiago Camargo Alves (tiago.camargoalves@gmail.com) - PPMEC, Universidade de Brasília Joao Vitor Quintiliano Silveiro Borges (j.quintiliano@gmail.com) - PPMEC, Universidade de Brasília Leandro Bruno Alves Caio (engleandrocaio@gmail.com) - PPMEC, Universidade de Brasília Andréa Cristina dos Santos (andreasantos@unb.br)-EPR, PPMEC, Universidade de Brasília

\section{RESUMO}

A Síndrome Respiratória Aguda Grave Coronavirus 2 (SARS-CoV-2) atingiu rapidamente o status de pandemia global em 2020 criando uma enorme demanda por equipamentos médicos e de proteção individual. A manufatura aditiva, neste contexto, tornou-se uma solução para aliviar as demandas do setor de saúde. Este artigo apresenta como as abordagens de manufatura lean e o DfAM (Design for Additive Manufacturing) auxiliaram na implementação de uma linha de fabricação de manufatura aditiva para produção de protetores faciais. A abordagem metodológica foi a pesquisa-ação, no qual discentes de graduação, pós-graduação, profissionais e pesquisadores juntaram esforços para produzir e entregar 27.000 protetores faciais. $\mathrm{O}$ artigo contribui na direção da implementação da manufatura aditiva para produção de pequenos lotes de peças finais e oferece espaço para discussões futuras quanto digitalização da produção.

Palavras chaves: Manufatura aditiva, Design for Additive Manufacture (DfAM), Manufatura Lean, Projeto de produto. 


\section{INTRODUÇÃO}

A manufatura aditiva (MA) pode ser compreendida como um novo tipo de indústria que utiliza máquinas controladas por computador para transformar diretamente projetos digitais do computador em produtos físicos. Isso inclui as tecnologias que utilizam métodos de deposição de materiais para o desenvolvimento de estruturas de duas ou três dimensões. (VOLPATO, 2017).

Nos últimos 30 anos, o uso das tecnologias de MA passou por transformações. Iniciou com aplicações para produção de protótipos e, hoje, a tecnologia passa a configurar como uma opção para produção de peças de produtos acabados (THOMPSON et al., 2016). Estudos realizados por Beltrami e Orzes (2021) apontam para o potencial de novos negócios para pequenas e médias empresas a partir do emprego da MA.

Contudo, Li et al. (2019), Pradel et al. (2018) apontam como barreiras para adoção da manufatura aditiva para produção de peças finais, a carência de conhecimento sobre as capacidades e limitações desta tecnologia. Mesmo sendo uma nova tecnologia, continua-se considerando as recomendações de soluções e os conhecimentos baseadas na manufatura tradicionais para projetar e produzir peças (GARCIA-DOMINGUEZ; CLAVER; VICTHOR, 2020) ou ainda desenvolvendo produtos por tentativa e erro (TAREQ ET AL., 2021).

O objetivo deste artigo é apresentar como abordagem do DfAM (Design for Additive Manufacturing) e a manufatura lean auxiliaram na implementação de uma linha de fabricação por manufatura aditiva para produção de protetores faciais.

\section{REVISÃO TEÓRICA}

\subsection{DESIGN FOR ADDITIVE MANUFACTURING (DFAM)}

Os avanços da MA estão trazendo novas possibilidades de projeto e paradigmas de produção. Embora muito trabalho seja necessário para trazer o DfAM (Design for Additive Manufacturing) à maturidade, as pequenas e grandes empresas continuam explorando adoção da MA para peças finais de produtos. O DfAM está ainda em desenvolvimento (THOMPSON et al., 2016). Seu escopo de atuação tem sido mais abrangente quando comparado as abordagens tradicionais de DfMA (Design for Manufacturing Assembly). 
Na Figura 1 é apresentado o "ciclo digital” da MA, este, geralmente traz resquícios de tentativas e erros, iniciando com a criação de modelo 3D, com uma abordam tradicional em CAD (Computer Aided Design), em que são então realizados ajustes na conversão dos arquivos no formato SLT (Standard Triangle Language) e nos parâmetros de configuração do equipamento de impressão. Além destes, os softwares atuais de simulação geralmente não possuem módulos precisos que permitam simular o ciclo digital da MA com exatidão.

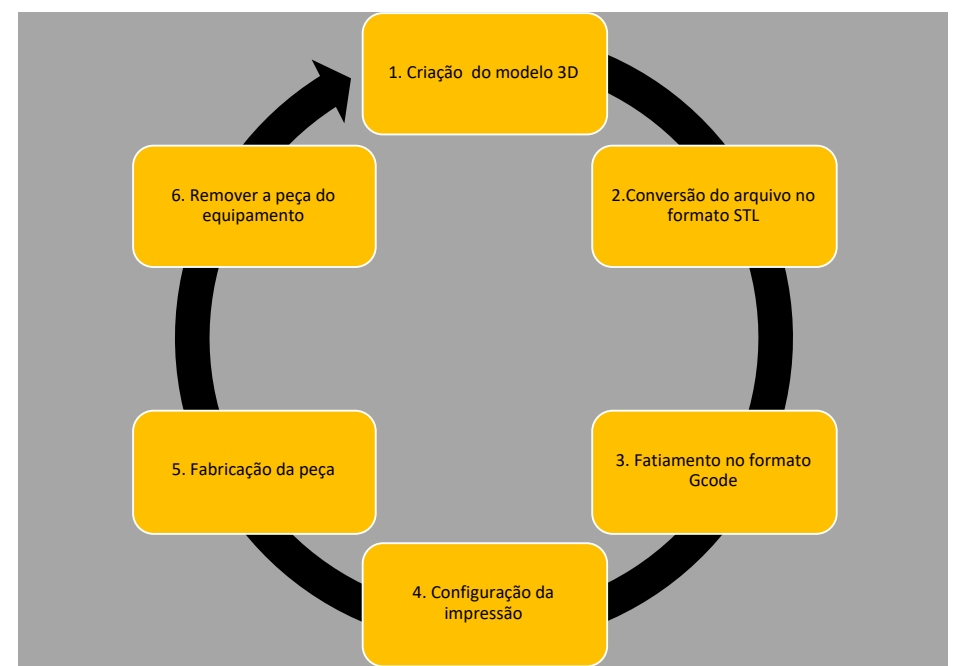

FIGURA 1 - Ciclo digital da MA. Fonte: Baseado em Pradel et al. (2018).

Diferentes modelos têm sido propostos com intuito de abordar o DfAM além do "ciclo digital". Entre eles se destaca os trabalhos de Pradel et al. (2018), Ponche et al. (2012), Boyard et al. (2014), Yang et al. (2015), Kumke et al. (2016) e Rosso et al. (2021). Estes levam em consideração todo o processo de desenvolvimento de produtos (PDP). Como exemplo, na Figura 2, apresenta-se o modelo de Pradel et al. (2018). Neste são apresentados diferentes fatores a serem considerados para a fabricação por MA, desde as fases iniciais do PDP.

Estudos mais aprofundados destes modelos revelaram que pouco é relatado sobre a fabricação de pequenos lotes. 


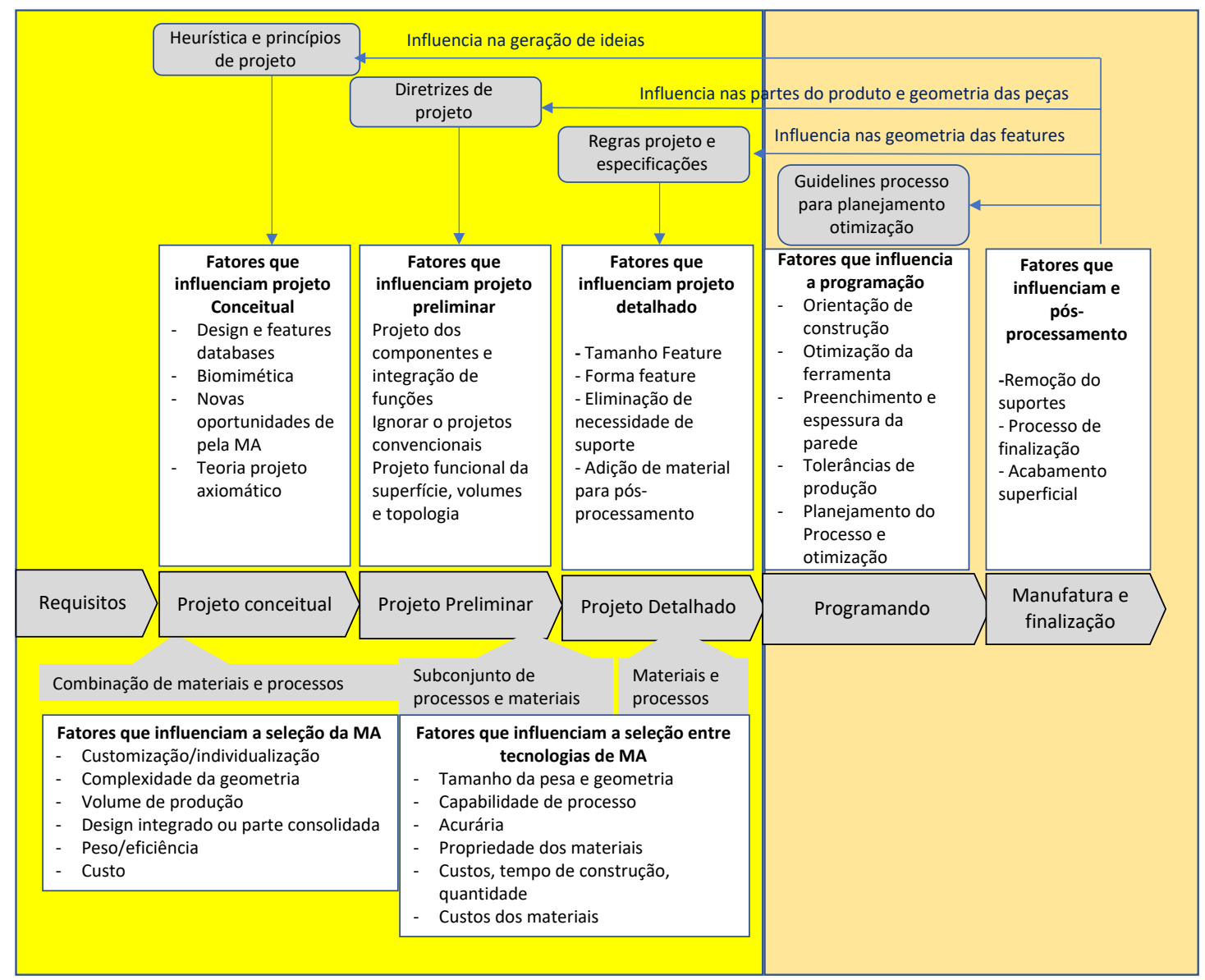

FIGURA 2 - Framework DfAM. Fonte: Pradel et al. (2018)

\subsection{OPORTUNIDADE DA MANUFATURA ADITIVA PARA A MANUFATURA LEAN}

De acordo com Womack e Jones (2005), a principal proposta da filosofia lean é otimizar de forma continua a entrega de valor para o cliente. A MA tem prometido revolucionar a Manufatura Lean (ML). Biazzo, Panizzolo e Gore. (2017) relacionam a importância do princípio do valor para MA, principalmente, na prototipagem para os clientes no contexto do processo de desenvolvimento. Ghobadian et al. (2018) colocam que a ML integrada a MA pode legitimar a eliminação dos desperdícios pela redução do tempo para o mercado; redução dos tempos de setup; redução das etapas de processamentos; redução dos requisitos por energia; melhora da qualidade das peças; redução da necessidade de retrabalho, melhora no acabamento das peças; possibilidade de fabricação de peças complexas. Além de possibilitar a redução dos custos totais de produção e encurtando a cadeia de suprimentos. O Quadro 1 apresenta as oportunidades da manufatura aditiva para manufatura lean. 


\begin{tabular}{|c|c|c|}
\hline Abordagem do Lean & Oportunidades para o Emprego da MA & Referências \\
\hline Negócio & $\begin{array}{l}\text { Produção sob encomenda em ambiente distribuído } \\
\text { Customização em massa de produtos. }\end{array}$ & $\begin{array}{l}\text { Khajari et al. (2014) } \\
\text { Gao et al. (2015) }\end{array}$ \\
\hline Projeto de produto & $\begin{array}{l}\text { Geometrias complexas; alterações e atualização podem ser } \\
\text { realizadas com maior facilidade. } \\
\text { Otimização de peso das peças a partir da liberdade de } \\
\text { fabricação de geometrias } \\
\text { Proporciona a manufatura de canais e estruturas internas } \\
\text { que aumentam a funcionalidade e desempenho dos } \\
\text { componentes } \\
\text { Acabamentos superfícies e texturas e porosidades } \\
\text { personalizadas }\end{array}$ & $\begin{array}{l}\text { Thompson et al. (2016) } \\
\text { Asadollahi-Yazdi et al. (2017) } \\
\text { Rosso et al. (2021) } \\
\text { Gibson et al. (2014). } \\
\text { Ponche et al. (2012) } \\
\text { Diegel et al. (2019) }\end{array}$ \\
\hline Manufatura & $\begin{array}{l}\text { Redução do tempo para o mercado; } \\
\text { Redução dos tempos de Setup; } \\
\text { Redução das etapas de processamento; } \\
\text { Redução dos requisitos de energia } \\
\text { Redução da necessidade de retrabalho e acabamento dos } \\
\text { componentes }\end{array}$ & $\begin{array}{l}\text { Oettmeier, Hofmann (2016) } \\
\text { Ghobadian et al. (2018) } \\
\text { Gibson et al. (2014). }\end{array}$ \\
\hline $\begin{array}{l}\text { Cadeia } \\
\text { suprimentos }\end{array}$ & $\begin{array}{l}\text { Reduzir custos de envio e redução dos prazos de entrega } \\
\text { Produzir peças sob demanda e assim reduzir a necessidade } \\
\text { de manter um inventário de segurança. } \\
\text { Encurtamento dos elos da cadeia de suprimentos }\end{array}$ & $\begin{array}{l}\text { Liu et al. (2014) } \\
\text { Ghadge et al. (2018) } \\
\text { Ghobadian et al. (2018) }\end{array}$ \\
\hline
\end{tabular}

Quadro 1 - Oportunidades para o emprego da Manufatura Aditiva para Manufatura Lean. Fonte: Autores

A ML se baseia no conceito que qualquer atividade/ação que falhe ao criar valor para os clientes é desperdício e deve ser reduzida (BIAZZO, PANIZZOLO, GORE, 2017). Logo, para que um sistema seja lean, ele deve abordar a redução da variabilidade nos relacionamentos na unidade de análise.

Pabliosa et al (2021) buscando integrar os conceitos da indústria 4.0 com a ML apresentam os principais métodos, técnicas e ferramentas para a operação (kanban, mapeamento do fluxo de valor, poka yoke, kaisen, produção puxada, Andon, SMED, padronização, TQM, takt time, heijunka, $5 \mathrm{~S}$, jidoka e TPM).

\section{METODOLOGIA}

A questão da pesquisa que norteou este trabalho foi como operacionalizar a produção de peças finais produzidas por MA. A origem desta pesquisa foi a produção dos protetores faciais via MA para enfrentamento da pandemia do COVID-19. Assim está pesquisa buscou contribuir na implementação da MA para produção de peças finais. 
A abordagem metodológica foi a pesquisa-ação, no qual discentes de graduação, pósgraduação, profissionais e pesquisadores juntaram esforços para produzir e entregar 27.000 protetores faciais no Distrito Federal. A justificativa da seleção do método foi devido a interferência no meio do estudo, com interação entre os pesquisadores e os envolvidos conduzida em tempo real.

As etapas para o desenvolvimento da pesquisa-ação foram o planejamento da pesquisa, coleta dos dados, análise dos dados, planejamento dos dados e ações, implementação, avaliação dos resultados e geração dos relatórios.

No ano de 2017 foi inagurado o Laboratório Aberto de Brasília - LAB na Faculdade de Tecnologia da UnB. No qual foram adquiridas impressoras 3D, com uma abordagem centrada na produção de protótipos. Em 2020, devido a Pandemia instalada pelo COVID_19 foi requisitado produção temporária em caráter emergencial para produção de protetores faciais.

A produção dos protetores faciais via MA para o enfrentamento da pandemia do COVID-19 foi realizada de forma emergencial e temporária, em todo o prédio da Unidade de Ensino de Graduação da Faculdade de Tecnologia da UnB, local este, que abriga o Laboratório Aberto de Brasília. A produção foi viabilizada porque todo o edifício foi fechado para as demais atividades acadêmicas, o que permitiu o uso de quantas áreas fossem necessárias.

$\mathrm{Na}$ etapa de planejamento, devido as características do projeto e tamanho da equipe, a abordagem de gerenciamento adotada foi baseada em Oppenheim (2004). Foi definido um líder de projeto, uma sala de projeto, a estrutura analítica com as entregas, o fluxo de trabalho e disponibilização e visualização dos dados e dos resultados gerados. A equipe foi composta por 4 discentes de pós-graduação e 30 alunos de graduação. Sendo que 4 dominavam o processo de MA e outros 10 foram treinados durante o projeto.

Durante o planejamento foi realizado uma exploração da literatura especializada, utilizada para o apoiar às decisões ao longo da pesquisa-ação. Parte desta atividade é apresentada na seção anterior.

As técnicas utilizadas para condução das fases da pesquisa ação envolveram: observações diretas, análises dos registros existentes, treinamentos, reuniões de planejamento, reuniões de execução de atividades, reuniões de análises dos resultados e elaboração de relatórios. 


\section{O PROJETO E A PRODUÇÃO DOS PROTETORES FACIAIS}

No início do projeto, fez-se o levantamento dos requisitos legais exigidos. Por ser um dispositivo para o setor de saúde, ele é regulado pela Agência Nacional de Vigilância Sanitária (ANVISA). Observa-se, mesmo com a flexibilização pela ANVISA da Norma RDC n.356, de 23/03/2020, em virtude da pandemia, havia uma remissão a outras normas que não foram flexibilizadas. Estas normas orientaram o projeto quanto aos fluxos gerais de produção, os pontos de inspeção e controle da qualidade, na criação do manual de qualidade do produto e o manual de boas práticas de fabricação.

Com base nos requisitos legais, no levantamento das necessidades dos profissionais do sistema de saúde e nas tecnologias disponíveis no LAB (Impressora tipo FDM) optou-se pela seleção de uma concepção validada internacionalmente, no caso, da empresa Prusa 3D, com sede na República Tcheca (PRUSA 3D, 2020).

Contudo, foi necessário revisar o projeto por completo. Na etapa de fabricação, os melhores resultados de qualidade da peça e tempo de impressão foram obtidos com uma única peça por máquina, utilizando como referencial teórico a abordagem de DfAM.

A Figura 3 apresenta o fluxo do processo de fabricação do produto. Nesta são apresentadas as entradas dos materiais: Filamento PLA, Placa de PETG, Rolo de Elástico, Papel Cirúrgico (Embalagem primária), Saco plástico (Embalagem 2 - secundária), Caixa de Papelão (Embalagem 3 - terciária) e Papel e tinta para rotulagem do produto. As etapas do processo de fabricação: Impressão 3D, raspagem (acabamento da peça) - Responsável pelo suporte da cabeça; Corte a Laser no qual se obtinha o visor seguido de uma etapa de lavagem e secagem; A etapa de corte do elástico. $\mathrm{Na}$ etapa de montagem, as partes eram integradas e montadas, formando o protetor facial produzido. 


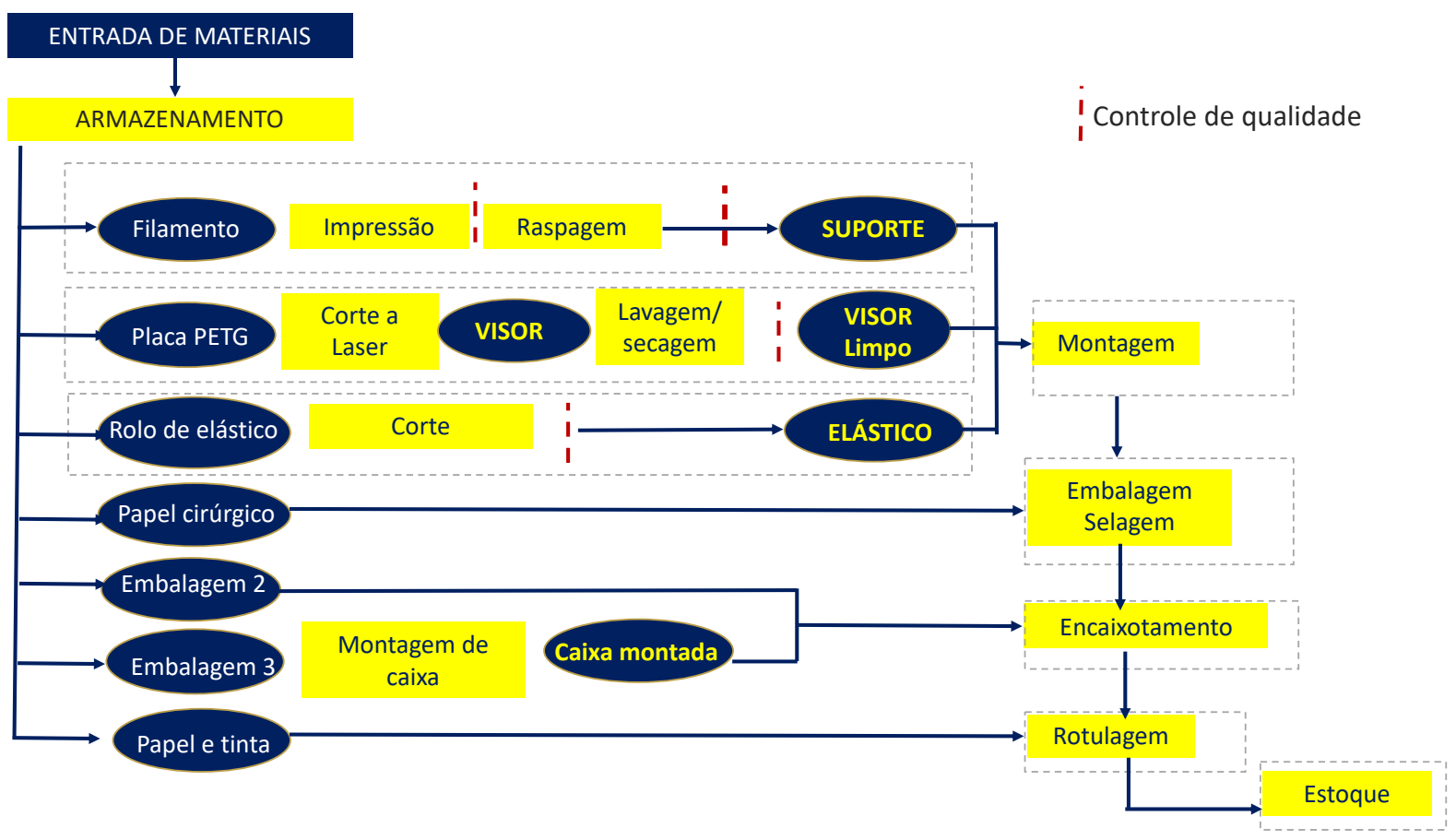

FIGURA 3 - Fluxograma de processo Fonte: arquivo do LAB (2020)

O projeto iniciou com apenas 7 impressoras, sendo realizado escalonamento da produção ao longo de 3 meses. Neste período foram adquiridas novas impressoras, sendo implementadas duas salas com 25 impressoras cada. A Figura 4 ilustra as salas de impressão.

A seleção da aquisição da tecnologia FDM foi em virtude da facilidade de manutenção dos equipamentos para ser realizada pelos próprios discentes.

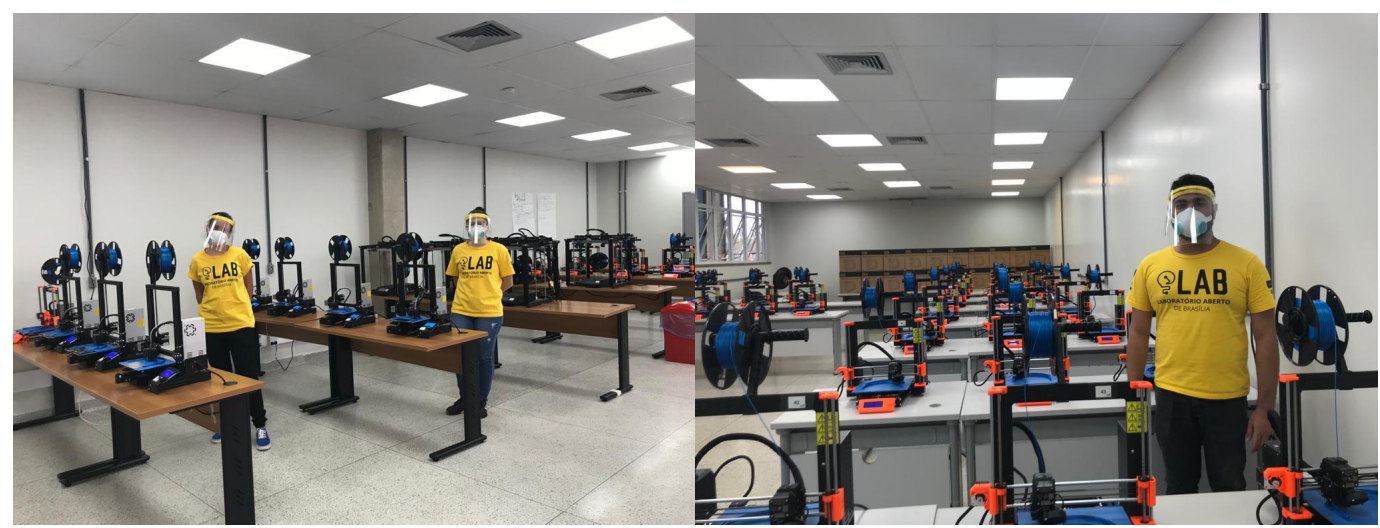

FIGURA 4 - Salas 1 e 2 de impressão Fonte: arquivo do LAB (2020) 
Um dos indicadores de controle de processo foi a disponibilidade de máquinas. A Figura 5 ilustra o monitoramento do processo de impressão. Com base dos dados do período de setembro de 2020 a janeiro de 2021, o indicador de disponibilidade de máquina foi de $98,3 \%$.

A partir da análise dos dados do período foi identificado que as paradas de máquinas ocorridas estavam associadas ao erro humano.
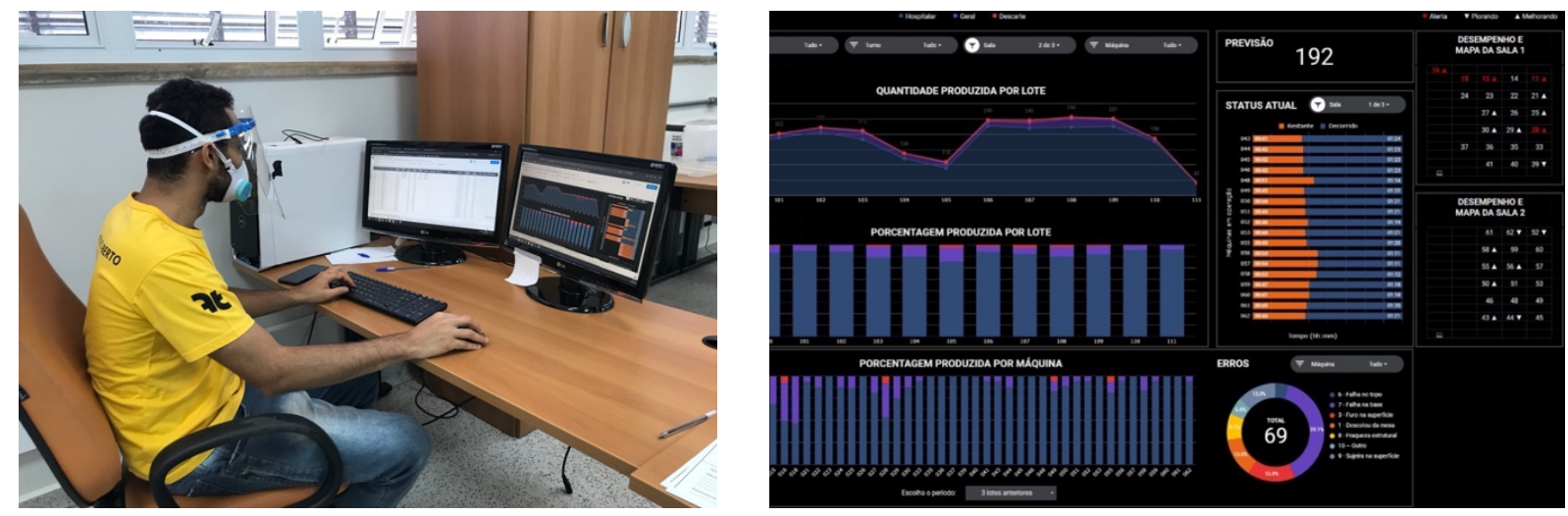

FIGURA 5 -Acompanhamento do processo de impressão 3D. Fonte: arquivo do LAB (2020)

Foi desenvolvido um sistema de rastreabilidade, com dados do processo produtivo, apresentado no Anexo A. A modelagem dos dados focou nos princípios para o gerenciamento em tempo real, interoperabilidade, virtualização, descentralização e agilidade e orientação ao serviço.

Neste projeto, em virtude da urgência e emergência, grande parte dos dados e controles de produto e processo foram realizados de forma tradicional. A base teórica sobre as possibilidades de digitalização dos dados foi introduzida nas discussões de reuniões e treinamentos da equipe. Como resultado obteve-se um conjunto de necessidades e requisitos para futuros projetos.

Outro indicador utilizado durante a fabricação foi o de ritmo de demanda (Takt Time). Para se manter a estabilidade no processo foram necessários a implementação de outras ferramentas da ML: o trabalho padronizado (os procedimentos operacionais padronizados - POP), 5S, Jidoka e o Kanban. Estas ferramentas foram selecionadas baseadas no tempo de operação da produção e a necessidade de capacitação em um curto período.

Além dos padrões operacionais descritos anteriormente, foram criados padrões visuais. $\mathrm{Na}$ Figura 6 são apresentados exemplos com diferentes tipos de anomalias na saída do processo de 
impressão.

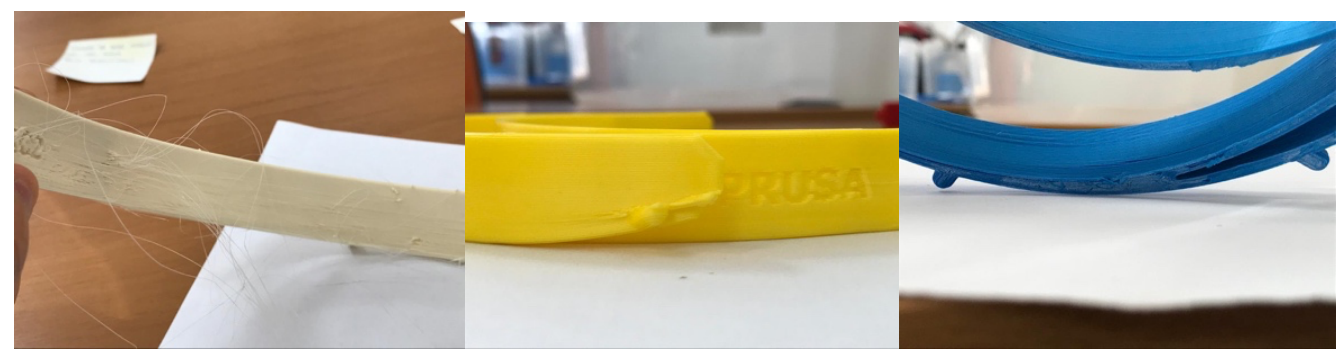

FIGURA 6 - Anomalias do processo impressão. Fonte: arquivo do LAB (2020)

Referente ao 5S, os espaços foram organizados com intuito de minimizar eventuais erros. Trabalhou-se com as ferramentas e materiais mínimos no local. Além da identificação visual.

Jidoka: Ao longo da fabricação foi exigido dos colaboradores uma análise detalhada dos potenciais defeitos dos itens. No início das operações foi natural o colaborador identificar um maior número de erros e com o tempo execução da atividade observou-se uma menor efetividade na identificação das falhas. Para minimizar a qualidade exagerada e a efetividade na identificação de potenciais falhas, foi realizada uma dupla checagem, a primeira referente aos itens reprovados e a segunda na liberação do item para próxima etapa de processo.

O Kanban foi utilizado em conjunto com o ritmo de produção, a equipe de projeto tinha informações como estava o andamento da produção e se mantinha atualizada sobre as metas diárias de produção. Além destes a estabilidade do processo de produção é dependente da estabilidade da mão de obra e da estabilidade de materiais:

A estabilidade de mão de obra exige que os colaboradores do projeto sejam altamente treinados com intuito de saber precisamente o que fazer, como fazer e quando fazer. Os treinamentos envolveram além de saber executar a atividade, buscaram o desenvolvimento de um senso crítico para melhoria da atividade que estava sendo realizada. Como exemplo temos a etapa de montagem dos protetores faciais. Ao longo do projeto foram montadas diferentes configurações do fluxo de montagem com intuito de otimizar os tempos de montagem e a quantidade de membros envolvidos nesta etapa. Ao final chegou-se uma configuração otimizada apresenta na Figura 7. 


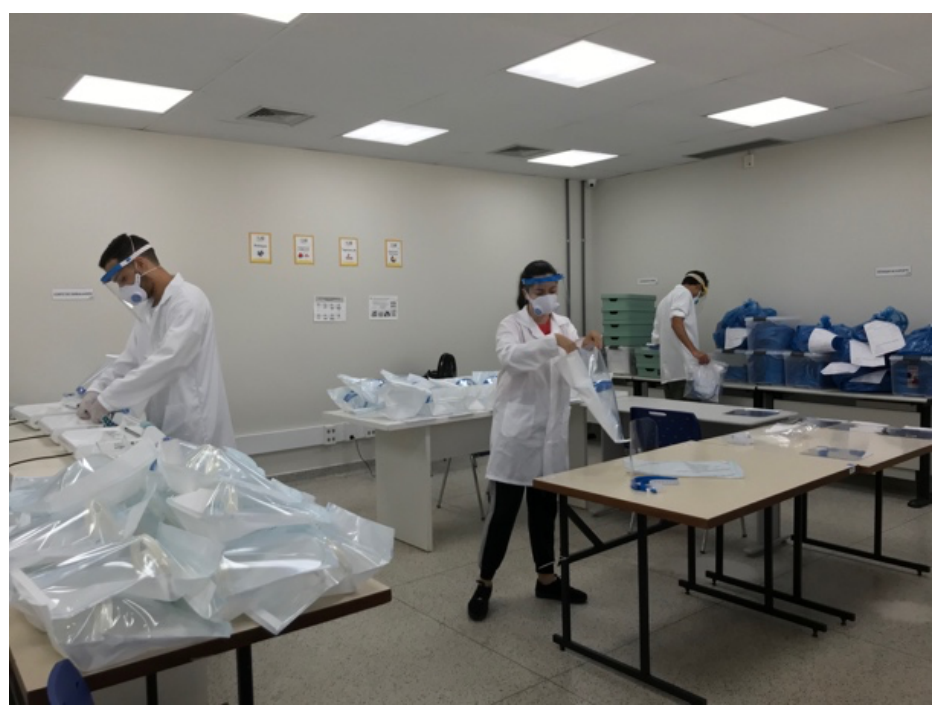

FIGURA 7 - Configuração da etapa do processo de montagem dos protetores faciais. Fonte: arquivo do LAB (2020)

A estabilidade dos materiais significa obter os materiais na quantidade e qualidade necessária nos postos de trabalho quando solicitados, evitando que a produção seja interrompida por falta ou defeito deles. Para tanto foi estabelecido um responsável pela movimentação do material para áreas. Este colaborador no início de cada turno conferia o material disponível no setor e fazia o abastecimento e coletava informações também sobre as perdas geradas na estação trabalho.

O desenvolvimento de novas versões do protetor facial teve como motivações reduzir os custos associados a etapa de fabricação e melhorar a qualidade do produto entregue aos usuários. No qual foram utilizados dados do Engenharia e Análise de Valor, testes de usabilidade e resistência mecânica. Os novos suportes desenvolvidos com menor massa e maior proteção da face obtiveram a redução de custos em $25 \%$ à versão original do projeto pela PRUSA $3 \mathrm{D}$. A Figura 8 apresenta as diferentes versões do produto.

Durante todo o projeto manteve-se uma conversa direta com os usuários do protetor facial, via Núcleos de Qualidade do Paciente dos Hospitais do DF. Esta comunicação teve como objetivo identificar possíveis melhorias no produto e realizar a gestão da produção e a demanda por protetores faciais para entrega. 

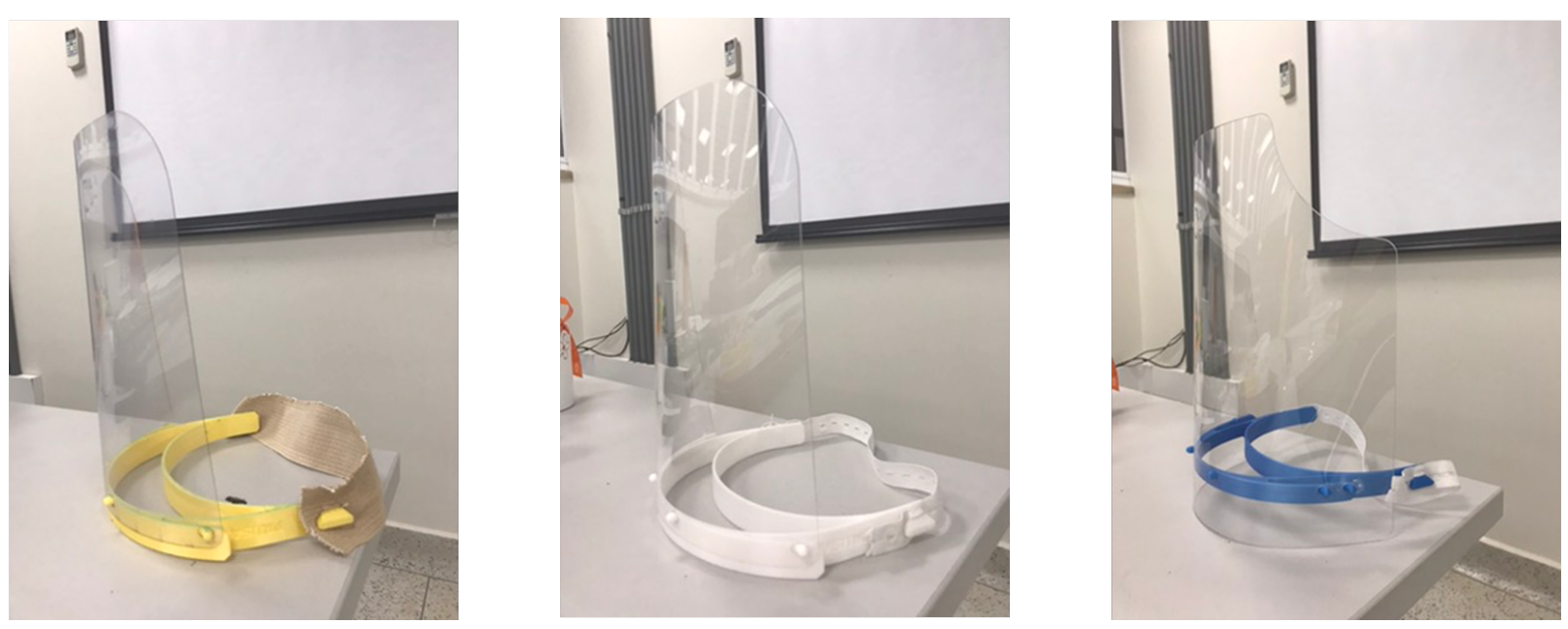

FIGURA 8 - Versões do protetor facial. Fonte: arquivo do LAB (2020)

Ao longo do projeto houve problemas no fornecimento de insumos, no primeiro momento devido aos constantes lockdowns e no segundo momento pela retomada da atividade das cadeias suprimentos as quais priorizarem clientes de mais longo prazo.

O Quadro 2 apresenta a síntese dos resultados obtidos pelo emprego do DfAM e Manufatura Lean na produção dos protetores faciais.

\begin{tabular}{|l|l|}
\hline \multicolumn{1}{|c|}{ Abordagem do Lean } & \multicolumn{1}{c|}{ Resultados/DfAM e ML } \\
\hline Negócio & - Tempo reduzido de inserção de entrega de novas versões do produto; \\
& - Facilidade de ampliação da escala de produção. \\
& - Implementação de uma linha de fabricação por manufatura aditiva de baixo custo
\end{tabular}

Quadro 2 - Síntese dos resultados obtidos com abordagem Lean e as oportunidades da MA. Fonte: autores 


\section{CONSIDERAÇÕES FINAIS}

Em virtude da pandemia instalada pelo COVID-19 foi requisitado ao Laboratório Aberto de Brasília (LAB), da Universidade de Brasília (UnB), a produção de protetores faciais, um dispositivo médico. O desafio inicial do projeto foi entregar um protetor facial para uso em ambiente hospitalar, no qual o cliente não estava disposto a esperar pelo desenvolvimento do produto, ou seja, a demanda era de produto pronto para o uso.

A partir dos feedbacks dos clientes e dos próprios problemas identificados na produção, corroborado pela integração da filosofia lean com o DfAM. As alterações e mudanças no projeto e na fabricação foram rapidamente implementadas, cerca de um dia.

Por meio do emprego da metodologia de pesquisa-ação, a equipe envolvida absorveu os métodos e ferramentas da abordagem da filosofia lean e do DfAM, compreendendo os motivos das tomadas de decisão durante todo o projeto.

Em virtude da urgência e emergência, grande parte dos dados e controles de produto e processo foram realizados de forma tradicional. Contudo, destaca-se que a modelagem dos dados foi realizada com intuito de fornecer subsídios para futuros projetos.

Observa-se a tecnologia de MA necessita estar integrada a outras tecnologias da indústria 4.0 com intuito de se alcançar todo o seu potencial. No entanto, os métodos e ferramentas da ML utilizados foram suficientes para manter a qualidade do protetor facial e manter a estabilidade do processo de fabricação. Infere-se que estes resultados foram alcançados devido o compromisso e engajamento dos colaboradores do projeto, princípios presentes na ML.

Com os resultados obtidos referentes os benefícios da MA, infere-se a importância da ML para adoção MA para produção de peças finais de produtos, principalmente para a implementação das estratégias de redução da cadeia logística de abastecimento e atendimento da demanda de produtos mais próximo do cliente.

A continuidade da pesquisa envolve digitalização dos serviços prestados por meio da produção de peças produzidas por Manufatura Aditiva no LAB, permitindo a criação de um ambiente de simulação para a formação de estudantes em nível de graduação e pós-graduação. 


\section{AGRADECIMENTOS}

Agradecemos a todos os envolvidos diretos e indiretos no projeto.

Este projeto foi financiado pela Fundação de Apoio do Distrito Federal (FAP/DF) no âmbito do convênio $\mathrm{n}^{\circ} .03 / 2020$, destinado ao combate do COVID-19, com a Fundação de Empreendimentos Científicos e Tecnológicos (FINATEC).

Este projeto recebeu recursos financeiros suplementares do Ministério Público do Trabalho.

\section{REFERÊNCIAS}

AGÊNCIA NACIONAL DE VIGILÂNCIA SANITÁRIA. Resolução - RDC n. 356, de 23 de março de 2020. 2020. Disponível em: http:/www.in.gov.br/en/web/dou/-/resolucao-rdc-n356-de-23-de-marco-de-2020249317437. Acesso em: 23 mar. 2020.

BELTRAMI, M., ORZES, G. Additive manufacturing applications perspectives in small and medium enterprises. Natural Science, n.2, 2021. DOI:10.12982/CMUJNS.2021.024

BIAZZO, S.; PANIZZOLO, R.; GORE, A. Lean product development implementation approach: empirical evidence from Indian lean manufacturing. International Journal of Industrial Engineering and Management. v.8, n.3, p.189-201, 2017.

BOYARD, N. et al. A design methodology for parts using additive manufacturing. In: 6TH INTERNATIONAL CONFERENCE ON ADVANCED RESEARCH IN VIRTUAL AND RAPID PROTOTYPING. [S.1.: s.n.], 2014. p. 399-404.

GARCÍA-DOMINGUEZ, A., CLAVER, J., SEBASTIAN, M. A. Integrating of additive manufacturing, parametric design and optimization of parts obtained by fused deposition modeling (FDM). A methodological approach. Polymers Multidisciplinary, v. 12, n.9, p.1933, 2020.

GHADGE, A. et al. Impact of additive manufacturing on aircraft supply chain performance: A system dynamics approach. Journal of Manufacturing Technology Management, v. 29, n. 5, p. 846-865, 2018.

GHODADIAN, A. et al. Examining legitimitization of additive manufacturing in the interplay between innovation, lean manufacturing, and sustainability. International Journal of Production Economics. p.457-468 2018. DOI: 10.1016/j.iijpe.2018.06.001

KUMKE, M., WATSCHKE, H., VIETOR, T. A new methodological framework for design for additive manufacturing. Virtual and Physical Prototyping, v. 11, n. 1, p. 3-19, 2016.

LI, L. et al. Multi-view feature modeling for design for additive manufacturing. Advanced Engineering Informatics. v. 39, p. 144-156, 2019.

LIU, P. et al. The impact of additive manufacturing in the aircraft spare parts supply chain: supply chain operation reference (SCOR) model-based analysis. Production planning \& control. v. 25, p. 13-14., 2014.

OETTMEIER, K.; HOFMANN, E. Impact of additive manufacturing technology adoption on supply chain management processes and components. Journal of Manufacturing Technology Management, v. 27, n. 7, p. 944968, 2016.

OLSEN, T. L.; TOMLIN, B. Industry opportunities and challenges for operations management. Manufacturing e Service Operations Managements. v.22, p.113-122, 2020.

OPPENHEIM, B. Lean product development flow. Systems Engineering, v.7, n.4, p.352-376, 2004.

PAGLiOSA, M.; TORTORELlA, G.; FERREIRA, J. C. E. Industry 4.0 and lean manufacturing. Journal of Manufacturing Technology Management. v.32, n.3, p. 543-569, 2021. 
PONCHE, R. et al. A new global approach to design for additive manufacturing: A method to obtain a design that meets specifications while optimizing a given additive manufacturing process is presented in this paper. Virtual and Physical Prototyping, v. 7, n. 2, p. 93-105, 2012.

PRADEL, P. et al. A framework for mapping design for additive manufacturing knowledge for industrial and productdesign, Journal of Engineering Design, v.29, n. 6, p.291-326, 2018

PRUSA 3D. Printed face shields for medics and professionals. Disponível em http://www.prusa3d.com/covid19. Acesso: 20 de abr. 2020.

ROSSO, S. et al. An optimization workflow in design for additive manufacturing. Applied Sciences, v.11, 2021. DOI: $10.3390 /$ app110662572

TAREQ, M. S. et al. Additive manufacturing and the COVID-19 challenges: An in-depth study. Journal of Manufacturing Systems, v. 1, 2021. DOI: 10.1016/j.jmsy.2020.12.021.

THOMPSON, M. K. et al. Design for additive manufacturing: trends, opportunities, considerations, and constraints. CIRP Annual -Manufacturing Technology, v. 65, p.737-760. 2016,

VOLPATO, N. (Org.). Manufatura Aditiva e aplicação da Impressão 3D. São Paulo: Blucher. 2017.

WOMACK, J. P.; JONES, D. T. Lean consumption. Harward Business Review, v.83, n.3, p.58-68, 2005.

YANG, S. et al. Towards an automated decision support system for the identification of additive manufacturing part candidates. Journal of Intelligent Manufacturing. v.31, p.1917-1933.

ZHU, Y. et al. An Improved density-based design method of additive manufacturing fabricated in homogeneous cellular-solid structures. International Journal of Precision Engineering and Manufacturing. v.21, n.1, p. 103-116, 2020 . 


\section{(13ำ Congresso Brasileiro de Inovação e Gestão de Desenvolvimento do Produto

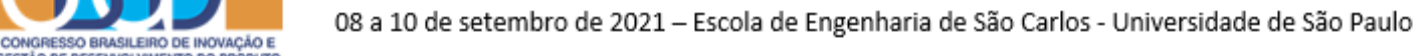

ANEXO A - Rastreabilidade dos itens

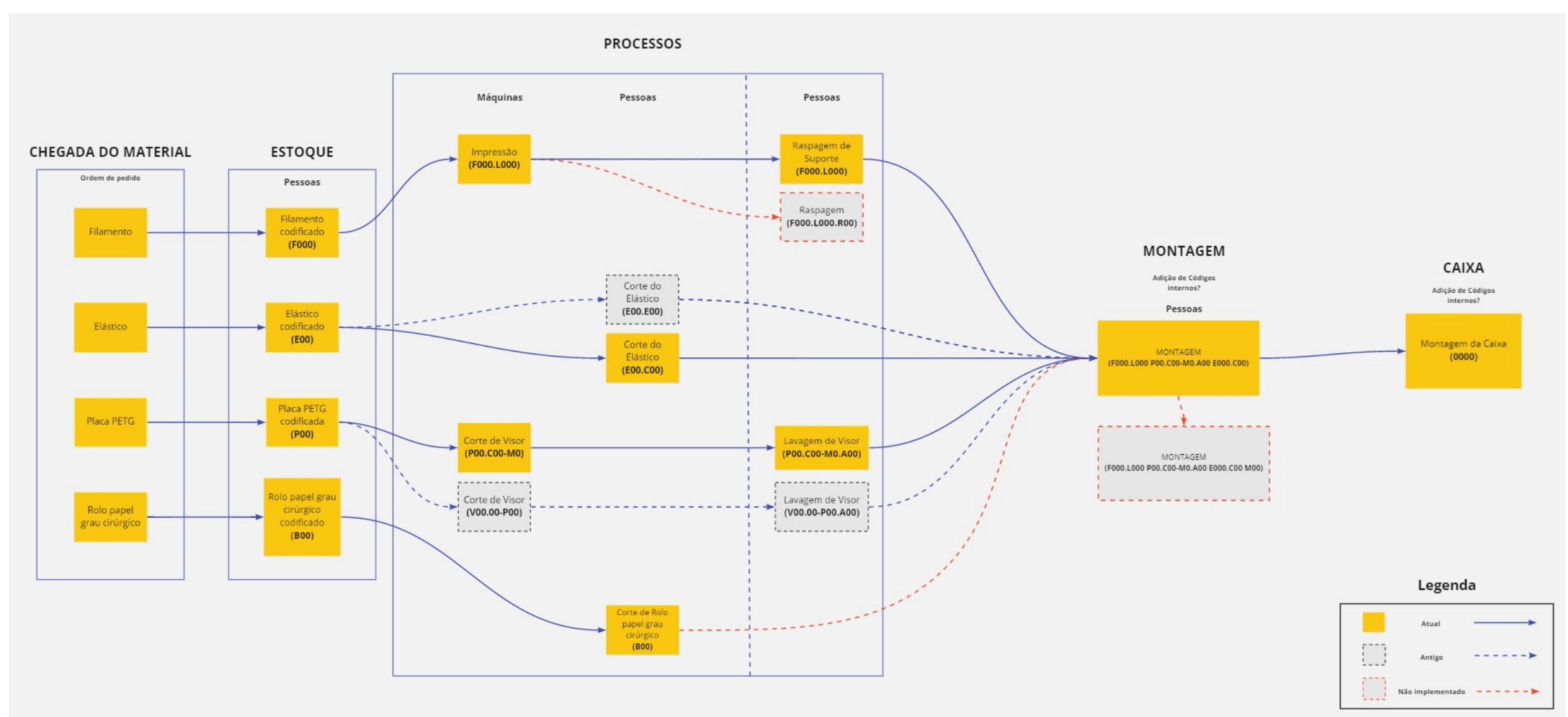

Fonte: arquivo do LAB (2020) 